\title{
Dispersive pressure and velocity fluctuations in avalanches-Reply to comment by $K$. Kelfoun and T. Davies on "A random kinetic energy model for rock avalanches: Eight case studies"
}

\author{
Perry Bartelt ${ }^{1}$ and Othmar Buser ${ }^{1}$ \\ Received 3 January 2011; revised 18 January 2011; accepted 21 January 2011; published 3 March 2011.
}

Citation: Bartelt, P., and O. Buser (2011), Dispersive pressure and velocity fluctuations in avalanches-Reply to comment by K. Kelfoun and T. Davies on "A random kinetic energy model for rock avalanches: Eight case studies", J. Geophys. Res., 116, F01015, doi:10.1029/2010JF001956.

[1] Mr. Kelfoun and Mr. Davies [Kelfoun and Davies, 2011] raise several interesting questions concerning the role of granular fluctuations in avalanche flow. Many of these comments center around the confusion of how and where granular fluctuations are created in rock avalanches, how they can produce dispersive pressures acting against the static weight of the flow mass (confining pressure), and how granular fluctuations influence avalanche mobility. Furthermore, Mr. Kelfoun and Mr. Davies question the comparison of rock avalanches to snow avalanches, the only geophysical flow where scientists have measurements from large (over 100,000 $\mathrm{m}^{3}$ ) real-scale flows consisting of hard ice granules in largely reproducible conditions [Gauer et al., 2007, Kern et al., 2009, Buser and Bartelt, 2009]. Finally, Mr. Kelfoun and Mr. Davies question the application of a physics-based model to historically constrained case studies and the challenges inherent in numerical parameter studies.

[2] First, we must address the "existence" of random kinetic energy $\mathrm{R}$ or fluctuations. Rock avalanches, like snow avalanches, start as bulk masses that, at first, move as rigid bodies. As they descend, the rigid body breaks-up, or fragments, into a flow mass consisting of granules of different shapes and sizes, varying from large blocks to smaller particles. Since the particles composing the flow mass are not all moving with the same speed, there exists a velocity component that differs from the mean or "rigid body" (center of mass) velocity of the avalanche. The relative motion of the fragments results in an elongation or longitudinal stretching of the flow mass that changes the flow density as spaces develop between the fragments. In our paper, we simply attribute the kinetic energy associated with the velocity deviations from the mean to an additional energy component in avalanche flow. Importantly, the deviations exist both in the direction of flow and perpendicular to the basal surface. This energy exists, because avalanches do not descend as a rigid block or as coherent, undeformed masses. The question is not whether it exists (it is a simple mathematical definition [see Brilliantov and Pöschel, 2004]) but, rather, how big is it and what influ-

\footnotetext{
${ }^{1}$ WSL Institute for Snow and Avalanche Research SLF, Davos, Switzerland.

Copyright 2011 by the American Geophysical Union. 0148-0227/11/2010JF001956
}

ence these fluctuations have on the flow. Thus, there are many fundamental questions that must now be resolved, such as how is this energy created and destroyed, is it concentrated near the basal surface or some other region in the avalanche, what is its relation to the fragmentation process, and how does it affect the mobility (the shear resistance) of the flow.

[3] To explore these questions we address the idea of "dispersive" pressure. Rock avalanche models invoke the idea of dispersive pressure [see Davies and McSaveney, 2002; Davies et al., 1999, 2010] or "acoustic fluidization" [see Collins and Melosh, 2003] to explain how the weight of the flow mass $\mathrm{N}$ is carried (or relieved) by the vertical pressure arising from the granular interactions. Note that granular interactions necessarily involve velocity fluctuations since, if all the particles were moving at the same speed, there would be no interactions and therefore no additional pressure. It is customary to reduce the normal stress $\mathrm{N}$ by the dispersive pressure $\mathrm{p}$ to find the flow resistance $\mathrm{S}$,

$$
S=\mu(N-p)
$$

where $\mu$ is the Coulomb (or Bayerlee) friction constant. This relation ties dispersive pressure (particle interactions), normal stress, and shear stress together.

[4] Let us consider this idea, which is fundamental to the ideas we present in the paper, in more detail. Consider Figure 1, which shows a rock mass $\mathrm{m}$ (per unit meter) sliding on a surface.

[5] At the start, the pressure at the bottom (which we can measure) is equal to the weight of the flowing mass $\mathrm{N}$. Then, because of the granular interactions, a dispersive pressure arises. The interactions can be collisional or without loss of sliding contact. All that is required is a velocity component not in the direction of the mean. The additional pressure acts everywhere, sideways, downward, and upwards, but upwards is the only direction a net acceleration can occur in the perpendicular component of $g$. This is Newton: for every action there is an equal and opposite reaction. If the weight of the avalanche and the dispersive pressure are not in equilibrium we must have a corresponding acceleration of our center of mass. Any dispersive pressure that lifts the mass upwards will exert an increasing pressure at the bottom $\mathrm{N}+\mathrm{p}$. If the pressure $\mathrm{p}=$ ma does not exist then the 


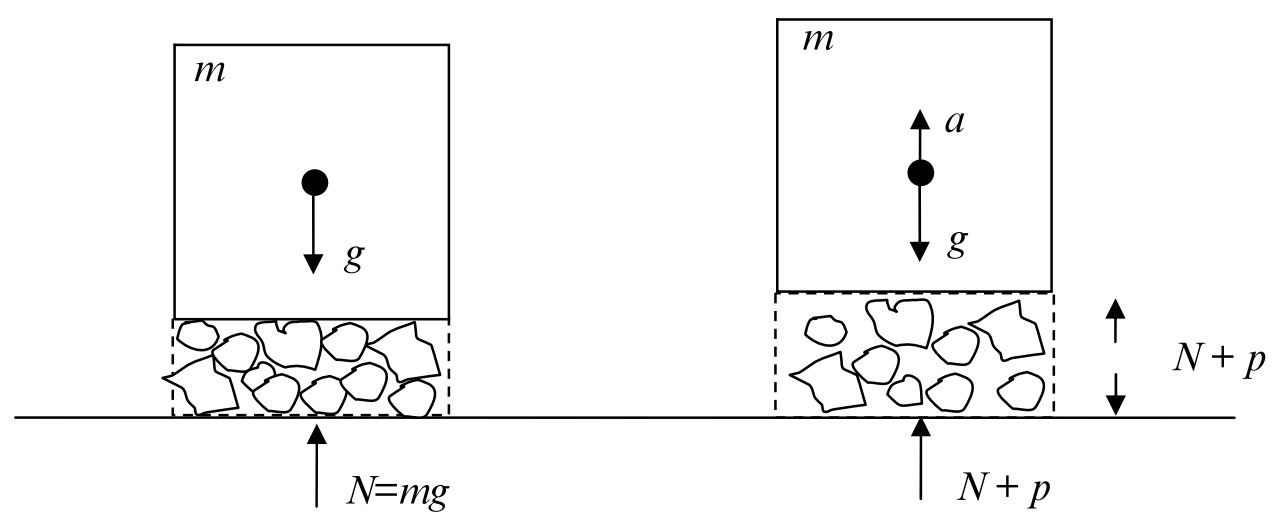

Figure 1. The concept of dispersive pressure applied to rock avalanches. If the pressure $p$ accelerates the mass upwards, there is a reaction on the bottom and the measured force is $\mathrm{N}+\mathrm{p}$, where $\mathrm{p}=$ ma.

mass is in equilibrium, and the force at the bottom is $\mathrm{N}$ for both rock avalanches and snow avalanches. Only when the center of mass is sinking (accelerating downward) will we measure $\mathrm{N}-\mathrm{p}$ at the bottom of the avalanche and "relieve" the overburden pressure. Gravity will oppose any upward acceleration so the mass will return, overshooting and oscillating around the equilibrium position in the absence of damping. Equation (1) is therefore an entirely empirical relation, with $\mathrm{p}$ an empirical parameter. (If the reader does not believe us, we suggest that he move up and down on his bathroom scale and look at the measured pressure.)

[6] A considerable source of confusion is the fact that Bagnold [1954] developed the concept of dispersive pressure for a system with a fixed (closed) volume. In a closed volume dispersive pressure can be maintained for a long time under shearing. This is not the case for avalanches, neither rock nor snow.

[7] If we allow the particle interactions to accelerate the center of mass upwards, we allow fluctuations to do mechanical work. We transform fluctuation energy into potential energy. The reason why the fluctuations can perform mechanical work (in an avalanche) is because of the basal boundary (defined where the velocities are zero) and the open boundary at the top surface of the avalanche. When this happens in shallow flows (several meters) such as snow avalanches, the flow height increases. The result is a decrease in flow density (the spacing between particles increases), especially at the avalanche head. In rock avalanches it is unlikely that the granular interactions can accelerate the mass upwards (because of the large overburden, here we agree); but this is exactly what we model in our paper: We do not allow the fluctuation energy to perform mechanical work: the flow density remains constant. Once more, even if the overburden is large, the pressure at the bottom is equal to $\mathrm{mg}=\mathrm{N}$ in the absence of an additional acceleration.

[8] In our analysis we have purposely considered ensemble averages (center of mass) of the particle movements. We do not consider individual particle trajectories. We do not pose the question of what is the effect of one collision or base our calculations on coefficients of restitution of single particles. As in gas dynamics we consider the macroscopic result of the numerous particle interactions on the whole. This leads us directly to the application of the principles of thermodynamics: energy conservation and the irreversibility of processes [Glansdorff and Prigogine, 1977]. Here lies one of the great challenges of granular avalanche dynamics: taken on the whole, the particle interactions both create fluctuation energy (particles with different speeds collide and are deflected); but these very same collisions dissipate energy (the collisions are certainly not elastic). Like accountants, we are confronted with a very delicate energy bookkeeping problem [Bartelt et al., 2006]. The particle fluctuations represent an intermediate energy form between the organized (mean) kinetic energy of the flow and terminal heat energy. Our energy bookkeeping must instruct us how to extract random kinetic energy from the mean flow, while likewise accounting for its dissipation. In the RKE model we create random energy $\mathrm{R}$ from the frictional shear rate of the mean flow (parameter $\alpha$ ). Other sources of $\mathrm{R}$ are possible, for example, the production of random energy by fracture as Mr. Kelfoun and Mr. Davies propose [Kelfoun and Davies, 2011]. Any energy released (exothermic reaction) or consumed (endothermic reaction) during the fragmentation process contributes to (source) or is taken from (sink) the fluctuation energy R. Why? Because the resulting energy flux due to fracturing has no preferential direction and is therefore random: there is no net acceleration in the flow direction. However, unlike fracture (which is a limited process), shearing provides us with a continual source for R.

[9] By making shear work the source of the random kinetic energy, we ensure that when the avalanche stops, the random kinetic has vanished. The decay constant $\beta$ describes how quickly the random energy disappears in the absence of a source (shearing, exothermic fragmentation). It accounts for all inelastic processes. Although it is related to the collisional properties of the fragments, it cannot be determined by considering a single collision or mean free path length, or by the energy consumed by a single fracture (endothermic reaction) as $\mathrm{Mr}$. Kelfoun and Mr. Davies calculate [Kelfoun and Davies, 2011]. Again, one must consider the ensemble average of the interacting granules. We assume the random energy is not dissipated in a single collision but on the sum of all the collisions, which is proportional to R. Again, we avoid the unsolvable problem of deterministically tracking an infinite chain of microscopic processes.

[10] Neglecting the velocity squared friction, our relation for shear is

$$
S=\mu(R)(N+p) \text { with } p=m a .
$$


Here, $p$ is the additional pressure from the upward acceleration, not an empirical parameter. Because of the large overburden, we assume for rock avalanches $\mathrm{a}=0$, and therefore $\mathrm{p}=\mathrm{ma}=0$. Again, the mobility given by $\mu$ is not a function of $p$. Thus, we distance ourselves not only from the ideas of Davies [Davies and McSaveney, 2002; Davies et al., 1999, 2010], but also from the proposition of Collins and Melosh [2003] where $\mathrm{p}$ arises from the "acoustic fluidization." For us these formulations are impossible because they violate Newton's law.

[11] However, we are uncertain of our functional relationship $\mu(\mathrm{R})$ for rock avalanches. We apply results from snow avalanches and granular materials [Bartelt et al., 2006, Buser and Bartelt, 2009] which show that $\mu$ evolves as a function of $\mathrm{R}$ from the head to tail of the avalanche. There is no reason to assume that physics is different in rock avalanches and granular avalanches (dense snow avalanches). The energy bookkeeping remains the same. Of course, the material properties change and therefore the model parameters change. This implies the model parameters depend on the material and terrain roughness. At present our production $(\alpha)$ is not constrained by the fragmentation process. It is $5 \%$ of the total shear work $(\alpha=0.05$, not $\alpha=5$ as Mr. Kelfoun and Mr. Davies correctly report in their comment [Kelfoun and Davies, 2011]). Fragmentation, if necessary, can be introduced within the framework of the RKE model by specifying the bulk random energy produced during fragmentation, as well as the energy consumed during fracture. This is unnecessary in snow avalanches where the fracture energy is small in comparison to the total potential energy of the avalanche.

[12] The substantial difference between rock and snow avalanches is admittedly the material properties, that is, the material constants. These material properties can all be included in the RKE model once one can formulate relations for the macroscopic energy fluxes. Because we have no direct measurements of particle size and velocity distributions, we use bulk material constants to account for the numerous microscopic events that occur in both snow and rock avalanches.

[13] We agree with Mr. Kelfoun and Mr. Davies that there is a need for "unambiguous and quantified field data" [Kelfoun and Davies, 2011]. However, it is unlikely that we will ever have such data for landslides and rock avalanches. For snow avalanches we have this possibility. They occur repeatedly on the same slope (sometimes two or three times in the same year), exhibit different flow characteristics depending on release size and granule properties. We have bulk measurements of shear and velocity [Gauer et al., 2007, Kern et al., 2009]. From our snow avalanche measurements, we have developed a model describing the movement of the avalanche from initiation to the postmortem state. We try now by induction to model the motion of rock avalanches, although we will never measure the internal dynamic behavior of the rockslide. Rather than placing demands on nonexistent flow data in rock avalanches, it is perhaps better to grasp the underlying physical processes and then take advantage of other fields where we have working models.

\section{References}

Bagnold, R. A. (1954), Experiments on a gravity-free dispersion of large solid spheres in a Newtonian fluid under shear, Proc. R. Soc. Lond. A, 225(1160), 49-63, doi:10.1098/rspa.1954.0186.

Bartelt, P., O. Buser, and K. Platzer (2006), Fluctuation-dissipation relations for granular snow avalanches, J. Glaciol., 52(179), 631-643, doi:10.3189/172756506781828476.

Brilliantov, N. V., and T. Pöschel (2004), Kinetic Theory of Granular Gases, Oxford Univ. Press, Oxford, U. K., doi:10.1093/acprof:oso/ 9780198530381.001.0001.

Buser, O., and P. Bartelt (2009), Production and decay of random kinetic energy in granular snow avalanches, J. Glaciol., 55, 3-12, doi:10.3189/ 002214309788608859 .

Collins, G. S., and H. J. Melosh (2003), Acoustic fluidization and the extraordinary mobility of sturzstroms, J. Geophys. Res., 108(B10), 2473, doi:10.1029/2003JB002465.

Davies, T. R. H., and M. J. McSaveney (2002), Dynamic simulation of the motion of fragmenting rock avalanches, Can. Geotech. J., 39, 789-798, doi:10.1139/t02-035.

Davies, T. R. H., M. J. McSaveney, and K. A. Hodgson (1999), A fragmentation-spreading model for long-runout rock avalanches, Can. Geotech. $J ., 36,1096-1110$, doi:10.1139/cgj-36-6-1096.

Davies, T., M. McSaveney, and K. Kelfoun (2010), Runout of the Socompa volcanic debris avalanche, Chile: A mechanical explanation for low basal shear resistance, Bull. Volcanol., 72, 933-944, doi:10.1007/s00445-0100372-9.

Gauer, P., D. Issler, K. Lied, K. Kristensen, H. Iwe, E. Lied, L. Rammer, and H. Schreiber (2007), On full-scale avalanche measurements at the Ryggfonn test site, Norway, Cold Reg. Sci. Technol., 49, 39-53, doi:10.1016/j.coldregions.2006.09.010.

Glansdorff, P., and I. Prigogine (1977), Thermodynamic Theory of Structure, Stability and Fluctuations, Wiley, London.

Kern, M., P. Bartelt, B. Sovilla, and O. Buser (2009), Measured shear rates in large dry and wet snow avalanches, J. Glaciol., 55, 327-338, doi:10.3189/002214309788608714.

Kelfoun, K., and T. Davies (2011), Comment on "A random kinetic energy model for rock avalanches: Eight case studies" by T. Preuth et al., J. Geophys. Res., 116, F01014, doi:10.1029/2010JF001916.

P. Bartelt and O. Buser, WSL Institute for Snow and Avalanche Research SLF, Flüelastr. 11, CH-7260 Davos, Switzerland. (bartelt@slf.ch) 